\title{
Pandemia covid-19 y calidad de vida en los emprendedores del sector gastronómico de la ciudad de HUANCAYO, 2021
}

Covid-19 pandemic and quality of life in entrepreneurs in the gastronomic sector of Huancayo city, 2021.

\author{
Giancarlo Giovanni Ponce Romero ${ }^{1, a}$, Roy River Vilca Rodríguez ${ }^{\text {1,b }}$, \\ Félix Simeón Castañeda Aranda 1,c, José Antonio Sánchez Meza 1,d,
}

\section{RESUMEN}

En el último año, todos los negocios se vieron afectados por la pandemia del COVID-19, al no poder desarrollar sus actividades habituales. Ocasionando en la mayoría de emprendedores diversos problemas. La investigación tiene como objetivo analizar la relación entre la pandemia COVID-19 y la calidad de vida en los emprendedores del sector gastronómico del distrito de Huancayo, 2021. Se empleó como material y método, el método científico con un enfoque cuantitativo y en razón del propósito del proyecto se aplicó el tipo descriptivo correlacional, la misma que tuvo como propósito el mostrar o examinar la relación que se tiene entre las variables de estudio. Dentro de los resultados obtenidos podemos afirmar con respecto a la hipótesis general que la misma fue aceptada debido a que el valor obtenido del coeficiente de correlación rho de Spearman $=-0.714$ evidenciando la existencia de una relación inversa media entre la pandemia COVID-19 y la calidad de vida en los emprendedores del sector gastronómico del distrito de Huancayo, 2021. Con respecto a las hipótesis especificas se establecen relaciones inversas medias de $-0.699,-0.674$ y -0.736 entre la variable independiente y las dimensiones de estudio de la variable dependiente (bienestar físico, bienestar emocional y desarrollo personal y profesional). Es dentro de este contexto que las conclusiones muestran que el principal desafío para todos los emprendedores de este sector, los mismos que ya se encuentran con una calidad de vida disminuida a causa de la pandemia ocasionada por la COVID-19, está en buscar la permanencia y desarrollo de sus organizaciones dentro de un mercado bastante adverso. La investigación concluye que existe una relación inversa media entre las variables de estudio.

PALABRAS CLAVE: pandemia COVID-19, calidad de vida, emprendedores.

\section{SUMMARY}

In the last year, all businesses were affected by the COVID-19 pandemic, unable to carry out their usual activities. Causing various problems in most entrepreneurs. The research has an objective to analyze the relationship between the COVID-19 pandemic and the quality of life in entrepreneurs in the gastronomic sector of the Huancayo district, 2021. The scientific method was used as a material and method with a quantitative approach and based on the For the purpose of the project, the descriptive correlational type was applied, the same one that had the purpose of showing or examining the relationship that exists between the study variables. Within the results obtained, we can

\footnotetext{
Universidad Privada de Huancayo Franklin Roosevelt. Huancayo, Perú

Docente; Economista; Magister. ORCID ID: 0000-0002-3502-4614

Docente; Lic. en Administración; Magister. ORCID ID: 0000-0002-0460-4341

Docente; Lic. en Administración; Magister. ORCID ID: 0000-0002-4664-1546

Docente; Lic. en Administración; Magister. ORCID ID: 0000-0002-8253-3198
} 
affirm with respect to the general hypothesis that it was accepted because the value obtained from Spearman's rho correlation coefficient $=-0.714$ evidencing the existence of a mean inverse relationship between the COVID-19 pandemic and the quality of life in entrepreneurs in the gastronomic sector of the Huancayo district, 2021. With respect to the specific hypotheses, mean inverse relationships of $-0.699,-0.674$ and -0.736 are established between the independent variable and the study dimensions of the dependent variable (physical well-being, emotional wellbeing and personal and professional development). It is within this context that the conclusions show that the main challenge for all entrepreneurs in this sector, the same ones who already have a diminished quality of life due to the pandemic caused by COVID-19, is to seek permanence and development of their organizations within a rather adverse market. The research concludes that there is a mean inverse relationship between the study variables.

Keywords: COVID-19 pandemic, quality of life, entrepreneurs.

\section{INTRODUCCIÓN}

El impacto de la pandemia ocasionado por la COVID-19 sobre la actividad económica dentro del país ha sido muy significativo, debido a las características de dicho impacto se pueden identificar tres fases vividas, inicialmente hablamos del cierre de varios países a finales del primer trimestre del año 2020 trayendo consigo la cancelación de vuelos dentro a nivel mundial, en segundo lugar el cierre de las actividades económicas dentro del país y una tercera fase enfocada a una lenta y gradual reapertura. En efecto, esta pandemia indudablemente puso en peligro la seguridad económica y la calidad de vida de las personas. En cuanto al impacto que se tuvo dentro de nuestro distrito, se puede afirmar que la pandemia trajo consigo una fuerte disminución dentro de la demanda interna, la cual arrastro consigo el desplome dentro de los niveles de producción.

El presente estudio centra su análisis de manera específica en los efectos que esta pandemia trajo a la calidad de vida de los emprendedores del sector gastronómico, según estadísticas del Ministerio de Comercio Exterior y Turismo (Mincetur), el sector gastronómico nacional mueve más de US\$ 5000 millones al año. En este sentido los emprendimientos privados del sector gastronómico del distrito de Huancayo que son los principales promotores dentro de su sector y la contribución que los mismos realizan dentro del tejido empresarial local permiten afianzarla como un instrumento de desarrollo y progreso. Salas (2020) sostiene que la gastronomía vive el peor momento de su historia reciente, la paralización total de esta actividad, además de generar mermas importantes, deja millonarias pérdidas económicas al sector. La
Cámara Nacional de Turismo del Perú (Canatur) señala que antes de la pandemia existían 200,000 restaurantes, que facilitaba empleo a más de 1 millón de personas a nivel nacional, pero de este total, el $50 \%$ de los negocios pequeños han tenido que cerrar, asimismo, la Asociación de hoteles, restaurantes y afines (Ahora Perú), informó que de marzo a mayo del 2020, se perdieron más de US\$ 700 millones en facturación, tomando en consideración solo a los establecimientos de comida formales. Los emprendimientos privados del sector gastronómico del distrito de Huancayo vienen afrontando una serie de retos de supervivencia para seguir operando dentro del mercado.

En cuanto al análisis de la calidad de vida, la misma involucra múltiples dimensiones interdisciplinarias, se trata de un constructo multidimensional, sus estudios han transitado las teorías del bienestar concebido como una situación de felicidad o satisfacción de deseos, placer y preferencias. Díscoli (2016) señala que el crecimiento económico no es una finalidad en sí mismo, sino un instrumento para crear mejores condiciones de vida. La calidad de vida está en función de las condiciones de vida y la satisfacción personal, medidas por una escala de importancia, en función de valores, aspiraciones y expectativas de la persona. En este sentido la Organización Mundial de la Salud [OMS] plantea que la calidad de vida es la percepción que tiene el individuo de su posición con respecto a la misma, según el contexto de su cultura y el sistema de valores en el cual vive, y en relación con sus objetivos, expectativas, normas e inquietudes (Botero y Pico, 2007). Para esta organización la calidad de vida se ve afectada por la salud física del individuo y su estado psicológico, grado de independencia, relaciones sociales las que son determinantes en el proceso de calidad de vida. 
Según Salazar (2020) la forma de abordar las diferentes estrategias que gestionen los riesgos vinculados a la pandemia COVID-19 influenciarán en la capacidad y el tiempo necesarios para una mejor recuperación económica, generándose a la vez una coyuntura única para afrontar los evidentes desequilibrios en igualdad, necesidades básicas vinculadas a la pobreza extrema, indigencia y exclusión social. Por tanto, es preciso establecer la influencia de la pandemia COVID-19 en la calidad de vida de los emprendedores del sector gastronómico, para emitir juicios valorativos sobre su imperio en la calidad y la satisfacción personal.

Santiago-Torner \& Rojas-Espinoza (2020) en su artículo titulado "Pandemia COVID-19 y calidad de vida. Relación dentro de una organización del sector eléctrico colombiano", el cual tuvo por objetivo analizar la relación de la pandemia COVID-19 y la calidad de vida de una organización del sector eléctrico colombiano. La metodología usada para este estudio fue con un enfoque cuantitativo con un diseño no experimental, transversal, correlacional y descriptivo. Los resultados identificaron una relación fuerte y positiva entre pandemia COVID-19 y la variable calidad de vida, es decir que las nuevas condiciones laborales establecidas por la urgencia sanitaria impactan en un $69 \%$ sobre la calidad de vida de las personas encuestadas. El estudio concluye que los cambios organizacionales producidos por la pandemia COVID-19 han optimizado la sensación de calidad de vida, como variable completa, de los trabajadores de la organización motivo de estudio mejorándose en un $73 \%$ desde que el trabajo es desde casa.

Samlani et al. (2021) en su artículo titulado "The impact of the COVID-19 pandemic on quality of life and well-being in Morocco". El cual mostró como objetivo de estudio evaluar la salud y el bienestar de los adultos no infectados con COVID-19 después de dos meses de cuarentena en Marruecos. Se realizó un estudio descriptivo transversal de 279 ciudadanos marroquíes por medio de una encuesta. Los resultados mostraron que la calidad de vida de todos los participantes se alteró moderadamente durante la pandemia COVID-19. La gravedad de la pandemia ocasionada por la COVID-19 tiene un impacto en la calidad de vida y el bienestar de la salud de las personas y este impacto es más marcado en personas con problemas de salud crónicos. La conclusión del estudio confirma la necesidad de prestar atención a la salud de las personas que no han sido infectadas por el virus. Las personas no infectadas con enfermedades crónicas pueden tener más probabilidades de tener problemas de bienestar debido a las restricciones de cuarentena.

Guzmán et al. (2021) en su artículo titulado "Impacto de un contexto de pandemia sobre la calidad de vida de adultos jóvenes", publicado en la revista cubana de medicina militar. En el cual se mostró como objetivo de estudio el comparar la calidad de vida auto reportada en un entorno sin pandemia vs. un contexto bajo alerta sanitaria por la COVID-19, en adultos jóvenes. El método aplicado fue un estudio retrospectivo en 2 situaciones: contexto sin pandemia y contexto con pandemia. Con un cuestionario de 8 ítems: función física, rol físico, dolor corporal, salud general, vitalidad, función social, rol emocional y salud mental. Los resultados que se observaron mostraron una disminución de la calidad de vida en las dimensiones vitalidad, función social, rol emocional, salud mental y salud general. Mientras que la dimensión más alterada fue rol emocional. La conclusión del estudio es que la calidad de vida relacionada con la salud de adultos jóvenes se ve disminuida en un contexto de cuarentena por la COVID-19, principalmente en las dimensiones psicológicas y sociales.

Villegas (2021) en su tesis titulada "Los efectos de la pandemia en pequeños emprendedores del sector de restaurantes en la ciudad de Guayaquil" presentada en la Universidad Católica de Santiago de Guayaquil Ecuador. La cual tuvo como objetivo el conocer los efectos de la pandemia sobre los emprendedores del sector restaurantes del norte de la ciudad de Guayaquil. La metodología utilizada para esta investigación es cualitativa con entrevistas a profundidad. Los resultados muestran que los emprendedores de restaurantes asumieron fuertes cambios e incluso muchos de ellos asumieron un nuevo comienzo debido a que los consumidores cambiaron y con ello sus principales necesidades. El estudio concluye que la pandemia fue una situación que provocó bastantes cambios en la vida de las personas, y que este tipo de negocios tuvieron que reinventarse cambiando su forma de comunicación y su servicio a domicilio.

Gonzales et al (2009) definen que la calidad de vida es el grado de bienestar emocional, social y físico en que se encuentra en una persona; así como la percepción y satisfacción individual de la vida dentro de una sociedad llena de valores creencias personales. Yataco (2008), refiere que, a lo largo del tiempo, el concepto de calidad de vida ha sido definido como la calidad de las condiciones de vida de una persona, 
como la satisfacción experimentada por la persona con dichas condiciones vitales, como la combinación de componentes objetivos y subjetivos, ponderadas por la escala de Valores, aspiraciones y experiencias personales.

La Teoría de los Dominios planteada por Olson y Barner (1982), citado por Grimaldo (2003) define a la calidad de vida como el fenómeno calidad de vida viene a ser un fruto de las relaciones entre las condiciones objetivas de vida y variables más subjetivas, personales, en una relación que da como resultado un mayor o menor índice de satisfacción y de felicidad de los individuos, es así que la calidad de vida viene a ser la medida compuesta por el bienestar físico, mental, social, tal como lo percibe cada individuo y cada grupo; como la felicidad, satisfacción y recompensa que puedan obtener.

Dentro de las dimensiones tomadas para determinar la calidad de vida; en primer lugar hablamos del bienestar físico, sobre el cual Mehech et al., (2016) nos dicen que es posible saber que la salud es un estado de bienestar en el que la persona conoce sus idoneidades, es capaz de vivir con las tensiones habituales del día a día, ayudar socialmente y ser productiva; por su lado García et al.,(2020) definen a la salud laboral como una actividad pluridisciplinaria que resguarda la salud de los empleados a través del control de accidentes y enfermedades disminuyendo los contextos de peligro. Morales (2013) explica que las organizaciones tienen la responsabilidad de proporcionar a sus colaboradores los medios necesarios para poder ejercer un mayor control sobre su vida y salud pudiéndolas así mejorar. No es una acción puntual sino una constante construcción colectiva, dinámica y participativa a favor del desarrollo y bienestar del ser humano, otorgando a los empleados un papel protagonista a través de espacios enmarcados en la construcción y transmisión de conocimiento junto con los recursos materiales y financieros necesarios para mejorar sus condiciones y estilos de vida derivándolos hacia la salud. La segunda dimensión viene a ser el bienestar emocional, el cual es visto como el equilibrio entre nuestros pensamientos, sentimientos y acciones teniendo en cuenta el vínculo existente entre lo que necesitamos y nuestra propia realidad junto con el respeto al sistema de creencias y valores que nos conforman.
Herrera et al., (2015) y Hirschle et al., (2019) demuestran que la regulación emocional, como elemento de control del estrés laboral, influye como estrategia adaptativa que resguarda la sensación de bienestar en el trabajo. La tercera dimensión viene a ser el desarrollo personal y profesional, los trabajos de Maslach y Jackson (1981) RodríguezGarcía et al. (2017) acercan a la relación entre desarrollo personal y Burnout como inductor de agotamiento emocional, despersonalización y una baja realización o desarrollo personal; al mismo tiempo (Galdeano y Barderas, 2010) indican que el establecer límites, la desconexión, ajustar las expectativas, la asertividad y el ampliar habilidades junto a nuevas perspectivas son elementos clave para evitar la falta de desarrollo personal a través de síntomas altos de desgaste emocional; asimismo Mondy et al., (2002); Bermúdez (2015) señalan que la capacitación es el centro del esfuerzo continuo para desarrollar y mejorar las capacidades de los colaboradores y de este modo su desempeño, los autores anteriormente citados también exponen que la capacitación es parte del desarrollo de las carreras individuales de los empleados y una función importante en la administración y planeación de los recursos humanos, la capacitación organizacional tiene otra función significativa y es motivar al trabajador para que sienta que puede desarrollar su capacidad creativa y que esta se relaciona con un mejor desarrollo personal y profesional; por su lado Chiavenato, (2009); Mehech et al., (2016) definen a la capacitación como un esfuerzo persistente para optimizar las competencias de los trabajadores y consecuentemente el desarrollo profesional.

Para el desarrollo de la investigación se planteó como problema general: ¿Cuál es la relación existente entre la pandemia COVID-19 y la calidad de vida en los emprendedores del sector gastronómico del distrito de Huancayo, 2021?, y tres problemas específicos: ¿Cuál es la relación existente entre la pandemia COVID-19 y el bienestar físico en los emprendedores del sector gastronómico del distrito de Huancayo, 2021?, ¿ Cuál es la relación existente entre la pandemia COVID-19 y bienestar emocional en los emprendedores del sector gastronómico del distrito de Huancayo, 2021?, ¿C Cuál es la relación existente entre la pandemia COVID-19 y el desarrollo personal y profesional en los emprendedores del sector gastronómico del distrito de Huancayo, 2021?. 
La investigación se justifica ya que a nivel de sociedad todos los empresarios desean poder salir del estancamiento al que hemos caído a causa de la pandemia ocasionada por la COVID-19, pero esto no será posible si primero no retoman aspectos fundamentales dentro de ellos mismos como personas y dentro de sus organizaciones, es importante conocer la relación entre la pandemia COVID-19 y la calidad de vida en los emprendedores del sector gastronómico, para de este modo poder apoyar al resurgimiento de este sector desde los mismos emprendedores identificando el bienestar con las condiciones de la persona tales como la capacidad, las oportunidades, las ventajas, un estado de felicidad y la libertad sobre los logros adquiridos por un individuo y las "capacidades" para poder lograrlo.

Como objetivo general: Analizar la relación entre la pandemia COVID-19 y la calidad de vida en los emprendedores del sector gastronómico del distrito de Huancayo, 2021. Y como objetivos específicos: Analizar la relación entre la pandemia COVID-19 y el bienestar físico en los emprendedores del sector gastronómico del distrito de Huancayo, 2021, Analizar la relación entre la pandemia COVID-19 y el bienestar emocional en los emprendedores del sector gastronómico del distrito de Huancayo, 2021 y Analizar la relación entre la pandemia COVID-19 y el desarrollo personal y profesional en los emprendedores del sector gastronómico del distrito de Huancayo, 2021.

La hipótesis general planteada dentro de la investigación afirma que existe una relación inversa entre la pandemia COVID-19 y la calidad de vida en los emprendedores del sector gastronómico del distrito de Huancayo, 2021. Planteando como hipótesis específicas: en primer lugar la existencia de una relación inversa entre la pandemia COVID-19 y el bienestar físico en los emprendedores del sector gastronómico del distrito de Huancayo, 2021, en segundo lugar la existencia de una relación inversa entre la pandemia COVID-19 y el bienestar emocional en los emprendedores del sector gastronómico del distrito de Huancayo, 2021 y por último se plantea la existencia de una relación inversa entre la pandemia COVID-19 y el desarrollo personal y profesional en los emprendedores del sector gastronómico del distrito de Huancayo, 2021.

\section{MATERIAL Y MÉTODOS}

De acuerdo con Carrasco (2005) "el método son los modos, las formas, las vías o caminos más adecuados para lograr objetivos previamente definidos" (Pág. 269). Para la investigación se empleó como método universal el método científico. Sobre el cual, Carrasco (2005) afirma que "el método, en tanto se emplea para realizar investigaciones científicas se denomina método científico, y constituye un sistema de procedimientos, técnicas, instrumentos, acciones estratégicas y tácticas para resolver el problema de investigación, así como comprobar la hipótesis científica” (Pág. 269).

La investigación en principio contó con un enfoque cuantitativo y en razón del propósito del proyecto se aplicó el tipo descriptivo - correlacional que según Bernal (2010, pág. 114) posee dentro de su esencia el "seleccionar las características fundamentales del objeto de estudio y su descripción detallada de las partes, categorías o clases de ese objeto" por lo que dentro del estudio se buscó desarrollar de forma más clara la relación existente entre la pandemia COVID-19 y la calidad de vida en los emprendedores del sector gastronómico de la ciudad de Huancayo, 2021. Para Salkind (1998, citado por Bernal 2010) la investigación de tipo correlacional busca como propósito el mostrar o examinar la relación que se tiene entre las variables de estudio o el resultado de variables, examinando las relaciones desarrolladas entre variables y sus resultados sin tratar de afirmar en ningún momento que una sea la causa de la otra. En otras palabras, la correlación examinará la asociación que tienen la pandemia COVID-19 y la calidad de vida en los emprendedores del sector gastronómico de la ciudad de Huancayo, 2021.

El nivel de investigación es descriptivo, cuyo propósito fue identificar las características, dimensiones, propiedades y regularidades del fenómeno que se encuentra en estudio. Según Schmelkes citado por Yarleque (2007). La presente investigación a este nivel buscó analizar la relación entre la pandemia COVID-19 y la calidad de vida en los emprendedores del sector gastronómico de la ciudad de Huancayo - 2021.

Las variables de estudio son: $\mathrm{X}$ (pandemia COVID-19) - Y (calidad de vida). 
La población que se consideró dentro del estudio estuvo comprendida por el total de empresas del rubro gastronómico inscritas ante la Superintendencia Nacional de Aduanas y de Administración Tributaria (Sunat), desde el año 2018 y que se encuentran operando a la fecha de elaboración de la investigación, las cuales suman un total de 720 organizaciones. Dentro del estudio se empleó un muestreo probabilístico, por el cual todos los miembros de la población presentaban la misma posibilidad de ser escogidos para formar parte de la muestra, de donde a través de cálculos estadísticos se obtuvo que la muestra estuvo comprendida por 251 emprendimientos de este rubro. Los instrumentos que permitieron acopiar las apreciaciones de los emprendedores para el desarrollo del estudio fueron dos cuestionarios diseñados con el objetivo de recoger información, el primero sobre la pandemia COVID-19 compuesto por 10 ítems, el segundo sobre calidad de vida compuesto por 15 ítems, mostrando un índice de fiabilidad de 0,752 y 0,8 obtenidos a través del alfa de Cronbach respectivamente.

\section{RESULTADOS}

A partir de los resultados obtenidos al momento de realizar la contrastación de las hipótesis planteadas, podemos aseverar con respecto a la hipótesis general que la misma fue aceptada debido a que el valor obtenido del coeficiente de correlación rho de Spearman $=-, 714 * *$ evidenciando la existencia de una relación inversa media entre la pandemia COVID-19 y la calidad de vida en los emprendedores del sector gastronómico del distrito de Huancayo, 2021. Con respecto a la primera hipótesis especifica, la misma fue aceptada debido a que el valor obtenido del coeficiente de correlación rho de Spearman $=-, 699 * *$ evidenciando la existencia de una relación inversa media entre la pandemia COVID-19 y el bienestar físico en los emprendedores del sector gastronómico del distrito de Huancayo, 2021. Con respecto a la segunda hipótesis especifica, la misma fue aceptada debido a que el valor obtenido del coeficiente de correlación rho de Spearman $=-, 674 * *$ evidenciando la existencia de una relación inversa media entre la pandemia COVID-19 y el bienestar emocional en los emprendedores del sector gastronómico del distrito de Huancayo, 2021. Con respecto a la tercera hipótesis especifica, la misma fue aceptada debido a que el valor obtenido del coeficiente de correlación rho de Spearman $=-, 736 * *$ evidenciando la existencia de una relación inversa media entre la pandemia COVID-19 y el desarrollo personal y profesional en los emprendedores del sector gastronómico del distrito de Huancayo, 2021.

\section{DISCUSIÓN}

Siendo el Perú uno de los países con la tasa más alta de emprendedurismo, como lo señala el Global Entrepreneurship Monitor. Perú 2018-2019, la ciudad de Huancayo es una urbe muy comercial y la pandemia por COVID-19 cambio drásticamente el panorama para el emprendedor especialmente para los del rubro gastronómico.

De acuerdo con los resultados de la investigación existe una relación inversa media entre la Pandemia COVID19 y la calidad de vida de los emprendedores del sector gastronómico de la ciudad de Huancayo, esto como es de entender se ve reflejado en que un $58.57 \%$ de los encuestados indican que la pandemia mostro un gran efecto y un $41,04 \%$ que mostró un efecto considerable sobre el descenso de su calidad de vida. Esto se contrasta con lo que sucede en otras industrias con mayor capacidad de adaptación, Borges de Amorim (2020) identifica más bien una relación fuerte y positiva entre pandemia COVID-19 y la variable calidad de vida en la industria eléctrica en Colombia, es decir que las nuevas condiciones laborales establecidas y los cambios organizacionales producidos han optimizado la sensación de calidad de vida de los trabajadores de la organización motivo de estudio mejorándose en un $73 \%$ desde que el trabajo es desde casa. En un contexto más similar Villegas (2021) analiza los efectos de la pandemia en pequeños emprendedores del sector de restaurantes en la ciudad de Guayaquil y evidencia que los emprendedores de restaurantes asumieron fuertes cambios e incluso muchos de ellos asumieron un nuevo comienzo debido a que los consumidores cambiaron y con ello sus principales necesidades, los emprendedores asumieron cambios en su vida y en sus negocios los que tuvieron que reinventarse cambiando su forma de comunicación y su servicio a domicilio, elementos que coinciden con los hallazgos de la presente investigación.

Definitivamente un sector como el gastronómico en la ciudad de Huancayo que es dirigido mayoritariamente por adultos jóvenes es impactado a nivel emocional y psicológico, Guzmán et al. (2021) comparan la calidad 
de vida auto reportada en un entorno sin pandemia vs. un contexto bajo alerta sanitaria por la COVID-19, en adultos jóvenes que evidenciaron una disminución de la calidad de vida en las dimensiones vitalidad, función social, rol emocional, salud mental y salud general. Siendo la dimensión más alterada el rol emocional, muy similares con los efectos en la calidad de vida que se encontraron en la presente investigación que muestra una percepción de afectación en su bienestar físico del $56,57 \%$, en su bienestar emocional del $56.97 \%$ y en su desarrollo personal y profesional del $54,18 \%$.

\section{CONCLUSIONES}

Este estudio se propuso analizar el entorno adverso, como el vivido durante la pandemia COVID-19y la calidad de vida en los emprendedores del sector gastronómico del distrito de Huancayo, es decir se estableció el valor obtenido del coeficiente de correlación rho de Spearman = ,$- 714 * *$ demostrando la existencia de una relación inversa media.

El análisis del COVID-19 y el bienestar emocional en los emprendedores del sector gastronómico la misma fue aceptada debido a que el valor obtenido del coeficiente de correlación rho de Spearman $=-, 674 * *$ evidenciando la existencia de una relación inversa media.

Por otro lado, al considerar la relación entre la pandemia COVID-19 y el bienestar físico en los emprendedores del sector gastronómico la misma fue aceptada debido a que el valor obtenido del coeficiente de correlación rho de Spearman = ,$- 699 * *$ evidenciando la existencia de una relación inversa media.

Con respecto a la pandemia COVID-19 y el desarrollo personal y profesional en los emprendedores del sector gastronómico del distrito de Huancayo la misma es aceptada debido a que el valor obtenido del coeficiente de correlación rho de Spearman $=-, 736^{* *}$ evidenciando la existencia de una relación inversa media.

En futuras investigaciones habría que plantear la posibilidad de repetir el estudio tras el final de la pandemia, con el fin de conocer en qué medida persisten o se han modificado los cambios detectados.

\section{Correspondencia:}

Giancarlo Giovanni Ponce Romero

Correo electrónico: gponce@uroosevelt.edu.pe

\section{REFERENCIAS BIBLIOGRÁFICAS}

1. Bermúdez, L. (2015). Capacitación: una herramienta de fortalecimiento de las pymes. Inter Sedes, 16(33), $1-25$.

2. Bernal, C. (2010). Metodología de la investigación: Administración, Economía, Humanidades y Ciencias Sociales. Pearson Educación.

3. Botero, B. \& Pico, M. (2007). Calidad de vida relacionada con la salud (CVRS) en adultos mayores de 60 años: una aproximación teórica. Revista hacia la Promoción de la Salud, 12, 11-24.

4. Carrasco, S. (2005). Metodología de la Investigación Cientifica. San Marcos.

5. Chiavenato, I. (2009). Gestión del talento humano. Mc Graw Hill.

6. Díscoli, C. (2016). Calidad de vida en el sistema urbano. Editorial Nobuko.

7. Galdeano, C. \& Valiente, A. (2010). Competencias profesionales. Educación química, 21(1), 28-32.

8. García-Escalera, J., Valiente, R., Sandín, B., Ehrenreich-May J. \& Chorot, P. (2020). Los efectos de un programa de prevención de la ansiedad y la depresión para adolescentes en variables educativas y de bienestar. Revista de Psicodidáctica, 25(2), 143149.

9. González, R., Freire, C., Piñeiro, I. \& González, P. (2010). Calidad de vida, bienestar y Salud. En R. González, A. Valle, R. Arce. \& F. Fariña. Calidad de vida, bienestar y Salud. Editorial Psicoeduca

10. Grimaldo, M. (2010). Calidad de vida y estilo de vida saludable en un grupo de estudiantes de posgrado de la ciudad de Lima. Pensamiento Psicológico, 8(15), $17-38$.

11. Guzmán,E., Concha,Y., Lira, C., Vásquez, J. \& Castillo, M. (2021). Impacto de un contexto de pandemia sobre la calidad de vida de adultos jóvenes. Revista Cubana de Medicina Militar, 50(2). http:// www.revmedmilitar.sld.cu/index.php/mil/article/ view/898/834

12. Herrera, L., Buitrago, R. \& Perandones T. (2015). Psicología Positiva e Inteligencia Emocional en Educación. Dedica. Revista de Educación e Humanidades, 8, 139-153.

13. Hirschle, A., Gondim, S., Alberton, G. \& Ferreira, A. (2019). Estresse e bem-estar no trabalho: o papel moderador da regulação emocional. Rev Psicol Organ Trab, 19(1),532-540.

14. Maslach, C., \& Jackson, S. (1981). The measurement of experienced burnout. Journal of organizational behavior, 2(2), 99-113. 
15. Mehech, C., Cordero, A. \& Gómez,T. (2016). Medición del compromiso laboral y su impacto en los resultados de la empresa. Universidad de Chile. https:// repositorio.uchile.cl/bitstream/handle/2250/139537/ Medici\%C3\%B3n\%20del\%20compromiso\%20 laboral $\% 20 \mathrm{y} \% 20$ su.pdf? sequence $=1$

16. Mondy, R., Noe, R. \& Premeaux, S. (2002). Human Resource Management. Prentice Hall.

17. Morales, L. (2013). El impulso lúdico: esencia y estructura del juego. Tonos digitales, 25, 1-27. https:// dialnet.unirioja.es/servlet/articulo? codigo $=4494845$

18. Salazar, F. (2020). Difusión de las Políticas Sociales: Diferencias entre los Programas de Lucha contra la Pobreza en Colombia y el Perú. [Tesis Doctoral, Pontificia Universidad Católica]. Repositorio Institucional de la Pontificia Universidad Católica del Perú. https://tesis.pucp.edu.pe/repositorio/handle/ 20.500.12404/16230

19. Rodríguez-García, A., Sola-Martínez, T. \& Fernández-Cruz, M. (2017). Impacto del Burnout en el desarrollo profesional del profesorado universitario. Una revisión de la investigación. Revista electrónica Interuniversitaria de formación del profesorado, 20(3), 161-178.

20. Salas, L. (2020). El trago amargo de la gastronomía peruana por el coronavirus y su largo camino a la recuperación. El Comercio.

21. Samlani, Z., Lemfadli, Y., Errami A., Oubaha, S. \& Krati, K. (2021). The impact of the COVID-19 pandemic on quality of life and well-being in Morocco. Archives of Community Medicine and Public Health.
22. Santiago-Torner, C. \& Rojas-Espinoza S. (2021). Pandemia COVID-19 y calidad de vida.

23. Relación dentro de una organización del sector eléctrico colombiano. Revista de Investigación, Desarrollo e Innovación, 11(3), 437-450.

24. Villegas, M. (2021). Los efectos de la pandemia en pequeños emprendedores del sector de restaurantes en la ciudad de Guayaquil. [Tesis de Título Profesional, Universidad Católica de Santiago de Guayaquil].Repositorio de la Universidad Católica de Santiago de Guayaquil. http://repositorio.ucsg.edu. ec/handle/3317/17268

25. Yarleque, C. (2007). Investigación en Educación y Ciencias Sociales. Ediciones Omega.

26. Yataco, A. (2008). Estadios de Cambio y calidad de vida en pacientes alcohólicos atendidos en los consultorios externos de farmacodependencia del Hospital Nacional Guillermo Almenara Irigoyen. [Tesis de segunda especialidad, Universidad Nacional Mayor de San Marcos] CYBERTESIS Repositorio de la Universidad Nacional Mayor de San Marcos. https:// cybertesis.unmsm.edu.pe/handle/20.500.12672/2507

Recibido: 04/06/2021

Aceptado: 18/11/2021 